\title{
Alismatis Rhizoma Triterpenes Regulate Metabolism of Renal Cells by AQP-2
}

\author{
Chao $\mathrm{Li}^{1,2 \dagger}$, Shi-Ting Pang ${ }^{1 \dagger}$, Zhi-Li Li ${ }^{1,3}$, Xing-Hua $\mathrm{Li}^{1}$, Qing-Lin $\mathrm{Lv}^{1}$, Liang $\mathrm{Ma}^{4 *}$, Liang Feng ${ }^{1,2^{*}}$ and Xiao-Bin $\mathrm{Jia}^{1,3^{*}}$ \\ ${ }^{1}$ School of Traditional Chinese Pharmacy, China Pharmaceutical University, Nanjing 211198, P. R. China \\ ${ }^{2}$ Jumpcan Pharmaceutical Co., Ltd., Jiangsu Taixing, 225400, P. R. China \\ ${ }^{3}$ College of Pharmacy, Jiangsu University, Zhenjiang 212013, P. R. China \\ ${ }^{4}$ Affiliated Hospital of Integrated Traditional Chinese and Western Medicine, Third Clinical Medical College, Nanjing \\ University of Chinese Medicine, Nanjing, Jiangsu, 210028, P. R. China \\ *For Correspondence: liangyu84@163.com; wenmoxiushi@163.com; jiaxiaobin2015@163.com \\ ${ }^{\dagger}$ Contributed equally to the work and are co-first authors \\ Received 23 April 2020; Accepted 05 October 2020; Published 10 January 2021
}

\begin{abstract}
The present research was planned to study the pharmacodynamic effects and mechanisms of terpenoids composition in Alisma orientale. In vivo test, the rat edema model was established with setting up the blank control group, the positive control furosemide group and high-dose, medium-dose and low-dose terpenoids groups. Collected rat urine after administration, and detected the biochemical indexes of rat urine. Detected the expression of medullary aquaporin 2 (AQP-2) in the rat kidney by Western Blot and immunohistochemistry and detected the expression of AQP-2 mRNA by polymerase chain reaction (PCR). In vitro test, using human tubular epithelial cell HK-2 model, the activity of HK-2 cells was determined with MTT colorimetric assay, the expression of AQP-2 protein in HK-2 cells was detected by Western Blot, the expression of AQP-2 mRNA was detected by RT-PCR, and IHC was used to detect the relative expression of AQP-2. In vivo test showed that terpenoids composition in alisma can increase urine volume, promote electrolyte excretion in urine and decrease the expression of AQP-2 in rat kidney. Results in vitro test showed that terpenoids composition cannot inhibit cells survival obviously, but reduce the relative expression of AQP-2. Conclusively, terpenoids composition in alisma inhibited the expression of AQP-2, having diuretic effect. (C) 2021 Friends Science Publishers
\end{abstract}

Keywords: Pharmacodynamic effects and mechanism; Alisma orientale; Terpenoids composition; Diuretic effect; HK-2 cells

\section{Introduction}

The renal collecting duct system contributes to the regulation of water reabsorption and final urine concentration (Mamuya et al. 2016). Aquaporin-2 (AQP-2), a channel protein located in the collecting duct, works in regulating urine concentration and maintaining electrolyte homeostasis in the body. It is regulated by arginine vasopressin, greatly improving water reabsorption in collecting tube (Jung and Kwon 2016). AQP-2 improves water permeability when transported to the apical membrane of the cell. The evidence showed that it can promote the reabsorption of water and the concentration of urine, reducing plasma osmotic pressure finally (Huang et al. 2018).

Alisma orientale (Sam.) Juzep (Alisma), a Chinese materia medica, has been used for thousands of years in China and other Asian countries. It has been found to hold water infiltration, heat release, lipid reduction and other bio-activities, and has been used for the prevention and treatment of adverse urination, full swelling of water, little diarrhea and urine, dizziness caused by phlegm, hot dripping and acerbity pain, hyperlipidemia. Modern phytochemical studies show that terpenoids are their main active ingredients, including triterpenes, sesquiterpenes and diterpenes (Jeong et al. 2016; Zhang et al. 2017a). In recent years, the accumulating evidence showed that alisma extract can significantly increase urine volume and increase the levels of $\mathrm{Na}^{+}, \mathrm{K}^{+}$and $\mathrm{Cl}^{-}$in rats (Zhang et al. 2017b). Studies have suggested that this regulatory activity may be closely related to AQP-2 (Mose et al. 2019). However, the effects of AQP-2 on the regulation of alisma on water-liquid metabolism remain unclear.

The research is aimed to study the roles of terpenoids from alisma in regulating urine concentration and maintaining electrolyte homeostasis and also explore the role of AQP-2 in this regulation and its mechanism. 


\section{Materials and Methods}

\section{Drugs and reagents}

Furosemide injection and TRIzol were respectively purchased from Wellhope Pharmaceutical Co., Ltd. (Shanghai, China) and Invitrogen (USA). cDNA first strand synthesis kit was purchased from ThermoFisher (USA). SYBR Premix Ex Taq II was purchased from TaKaRa Japan Company. Agarose I was purchased from Amresco (USA). All protein extraction kit, Braford protein content detection kit, SDS-PAGE gel preparation kit, pre-stained protein molecular weight, ECL detection kit, developing and determining reagents were purchased from Beyotime Biotechnology Co., Ltd. (Nantong, China). PVDF membrane was purchased from Merckmillipore Company (Germany). Rabbit beta-actin and AQP2 antibodies were purchased from BOSTER Biological Engineering Co., Ltd. (Wuhan, China). Secondary antibody (sheep anti-rabbit IgG, sheep anti-rat $\mathrm{IgG}$ ) was purchased from Abcam Company. Immunohistochemical kit was purchased from Hongshan Jinqiao Biotechnology Co. (Beijing, China). DMEM hyperglycemic-f12 1:1 incomplete medium, Dimethyl sulfoxide (DMSO) and FBS were respectively purchased from Corning (USA), Amresco and Gibco (USA). Tetramethylazoles salt (MTT) was purchased from Sigma Company (USA). Other reagents are commercial and analytical grade.

\section{Chromatographic conditions}

Spusil C18 chromatographic column $(150 \times 4.6 \mathrm{~mm}, 5$ micron) was used. Mobile phase: $(0.1 \%$ formic acid acetonitrile (95:5)) - $(0.1 \%$ formic acid - acetonitrile (15:85)); gradient elution (0-10 min, $100-40 \% \mathrm{~A} ; 10-25$ min, 40-0\% A; 25-35 min, 0\% A; 35-36 min, 0-100\% A; 36-48 $\mathrm{min}, 100 \% \mathrm{~A})$. The flow rate was $1.0 \mathrm{~mL} / \mathrm{min}$ and column temperature was $30^{\circ} \mathrm{C}$.

\section{Test products preparation}

The extract $(1 \mathrm{~mL})$ was taken and diluted with pure water to $5 \mathrm{~mL}$ for test.

Dry powder $(0.5 \mathrm{~g})$ was taken and put into a conical bottle with plug, $25 \mathrm{~mL}$ of $50 \%$ acetonitrile was added into the conical bottle accurately and treated with ultrasonic for 4 h. Then the extraction was filtered and the filtrate was taken for further experiments.

Dry powder $(0.5 \mathrm{~g})$ was taken and put into a conical bottle with plug, $25 \mathrm{~mL}$ of pure water was added into the conical bottle accurately and treated with ultrasonic for $4 \mathrm{~h}$. Then the extraction was filtered and the filtrate was taken for further experiments.

\section{Alisma terpenoids extraction and preparation}

Alisma terpenoids was prepared according to the following operation: alisma decoction pieces of $15 \mathrm{~kg}$ were weighed, crushed, and then extracted by reflux with 10 times volume $80 \%$ ethanol (v:v) for $30 \mathrm{~min}$. This extraction was carried out twice to obtain the filtrates for combination. The combined extraction was concentrated to about $0.5 \mathrm{~g} / \mathrm{mL}$ under decompression in a rotary evaporator. AB-8 macroporous resin column was used to purify the concentrated extract until the macroporous resin completely adsorbs. After removing impurities with $4 \mathrm{BV} \mathrm{50 \%} \mathrm{ethanol,} \mathrm{the} \mathrm{column}$ was eluted with $4 \mathrm{BV} 70 \%$ ethanol at speed of $0.3 \mathrm{BV} / \mathrm{h}$. The eluent was obtained with $70 \%$ ethanol and concentrated to obtain alisma terpenoids ( $125.03 \mathrm{~g}$, yield $0.83 \%)$.

\section{Animals and drug administration}

Male Sprague Dawley (SD) rats weighing $200 \pm 20 \mathrm{~g}$ were purchased from Nantong University, China (license number: SCXK (Su) 2014-0001), in line with SPF grade experimental animal standards. The SD rats were raised in the animal center of China Pharmaceutical University with room temperature $22-23^{\circ} \mathrm{C}$, humidity $45-50 \%$ and diurnal illumination of $12 \mathrm{~h}$ of light/12 h of darkness (L/D) cycles. Rats received food and water ad libitum. This study was approved by experimental animal ethics committee, and all experiments were in line with the guiding principles of animal research of Chinese ethics committee. Qualified model rats were screened by Aston method. These rats were raised adaptively in the metabolic cage for 3 days and then fasted for $8 \mathrm{~h}$ on fourth day. Sequentially, these rats were treated with $2.5 \mathrm{~mL} / 100 \mathrm{~g}$ distilled water, and urine was quickly collected for $2 \mathrm{~h}$ after administration. Rats whose urine volume were more than $40 \%$ gavage volume were considered qualified rats and randomly divided into blank control group ( $2.5 \mathrm{~mL} / 100 \mathrm{~g}$ distilled water); positive control group (furosemide of $20 \mathrm{mg} / \mathrm{kg}$ ); high-dose alisma terpenoids group (equivalent to decoction pieces $10 \mathrm{~g} / \mathrm{kg} / \mathrm{d}$ ); medium-dose alisma terpenoids group (equivalent to decoction pieces $5 \mathrm{~g} / \mathrm{kg} / \mathrm{d}$ ); low-dose alisma terpenoids group (equivalent to decoction pieces $2.5 \mathrm{~g} / \mathrm{kg} / \mathrm{d}$ ). The rats were administrated by $\mathrm{Chao} \mathrm{Li}$. The rats were treated with distilled water continuously for $3 \mathrm{~d}$ and fasted for $8 \mathrm{~h}$ before the last dose. Then rats in each group were given with $0.9 \%$ sodium chloride solution at the dose of $5 \mathrm{~mL} / 100 \mathrm{~g}$ to simulate water and sodium retention.

\section{Urine biochemical indicators detection}

After drug administration, the urine of rats were collected in a metabolic cage for $24 \mathrm{~h}$ and quantified accurately. The contents of $\mathrm{Na}^{+}, \mathrm{K}^{+}$and $\mathrm{Cl}^{-}$in the urine were detected by automatic biochemical analyzer (BS-490, Mindray, China) according to manufacturer's protocols.

\section{Western blotting}

The kidney tissues or HK-2 cells were added to the lysis 
buffer and ground at low temperature. After lysed, the tissues were centrifuged at $13000 \mathrm{rpm}$ at $4^{\circ} \mathrm{C}$ for $10 \mathrm{~min}$. Then the supernatant was taken for the further treatment. After the protein was denaturated at $100^{\circ} \mathrm{C}$, the same amount of total protein was separated by SDS-page electrophoresis and transferred to PVDF. The concentrated glue was kept at a constant pressure of $85 \mathrm{~V}$ for about 30 min, and the separated glue was kept at $120 \mathrm{~V}$ for about 80 min. The film transfer time was kept at $50 \mathrm{~min}$ with a constant current of $200 \mathrm{~mA}$. PVDF membrane was sealed with 5\% skim milk overnight and then added with Diluted primary antibody (1:1000). After shaking at $4^{\circ} \mathrm{C}$ overnight, PVDF membrane was washed with TBST twice (10 min for each time), and was washed with TBS for $10 \mathrm{~min}$. Secondary antibody diluent was added into the PVDF membrane and incubated at room temperature for 1-2 h. After PVDF membrane washed repeatedly with TBST solution and TBS solution, chemiluminescence was performed. Gel-pro 32 software was used to analyze the results in grayscale.

\section{Real-time quantitative polymerase chain reaction (q- PCR) analysis}

Kidney tissues or HK-2 cells were added into precooled TRIzol. The samples were grinded at low temperatures until split completely then transferred to PCR tube. Chloroform was added into tube and shook for 15s. After standing at $37^{\circ} \mathrm{C}$ for $10 \mathrm{~min}$, PCR tube was centrifuged at $12000 \mathrm{rpm}$ at $4^{\circ} \mathrm{C}$ for $10 \mathrm{~min}$. The residues were dissolved with $50 \mu \mathrm{L}$ RNase, and stored at $-70^{\circ} \mathrm{C}$ for later use. Sequentially, concentration and purity of RNA was determined. The primer was designed by Nanjing Jinsirui technology Co., Ltd, $\quad \beta$-actin $(175 \mathrm{bp})$ : upstream primer 5'GTGCTGAGTATGTCGTGGAGTC-3'; downstream primers 5'-TTGCTGACAAT CTTGAGGGA-3'; AQP2(132 bp): upstream primer 5'CCCTCTCCATTGGTTTCTCTGT TA-3'; downstream primers 5'-AGAAGACCCAGTGATCATCAAACTT-3'. The cDNA was synthesized using cDNA first strand synthesis kit. The PCR protocol was performed according to the procedure for PCR reaction: pre-denaturizing for $5 \mathrm{~min}$, denaturizing and annealing for $15 \mathrm{~s}$ at $95^{\circ} \mathrm{C}$ successively and developing for $60 \mathrm{~s}$ at $60^{\circ} \mathrm{C}$. The reaction without cDNA was included as a negative control. The date was analyzed by $2^{-} \triangle \mathrm{Ct}$ method.

\section{Immunochemistry}

The $4 \%$ formaldehyde solution was used to fix the rat kidney tissues for $24 \mathrm{~h}$ then the tissues were embedded with paraffin and cut into sections. The tissue was baked at $60^{\circ} \mathrm{C}$ for $1 \mathrm{~h}$ before dewaxing and immersed into xylene twice to dewaxing, and dehydrated in different gradients of alcohol $(70,85,95$ and $100 \%)$ for $5 \mathrm{~min}$. Sections were placed in antigen repair buffer, boiled for $20 \mathrm{~min}$ and PBS was used to wash the sections for 3 times. The sections were dripped with $3 \% \mathrm{H}_{2} \mathrm{O}_{2}$-PBS solution, then sealed for $15 \mathrm{~min}$ at room temperature and PBS was used to wash sections for 3 times. Primary antibody was added on sections $\left(4^{\circ} \mathrm{C}\right.$, overnight $)$ and washed with PBS for 4 times. Secondary antibody was added on sections and incubated for $30 \mathrm{~min}$ at $37^{\circ} \mathrm{C}$, then washed with PBS for 3 times. Control incubation was included with the secondary antibody alone. Appropriate amount of DAB solution was added on sections for staining, and color development was stopped with distilled water. The sections were redyed with sappanin dyeing solution for 10 min and immersed and dehydrated in different gradients of alcohol (70, 85, 95 and 100\%) for $5 \mathrm{~min}$. Then the sections were immersed into xylene twice. After the sections drying, neutral gum was added on the section for sealing. Negative controls were incubated and processed identically with omission of the primary antibody. The brown deposition was observed under a microscope.

\section{Cell culture and components treatment}

Human tubular epithelial cell line HK-2 was purchased from Nanjing KeyGEN Biological Development Co., Ltd. Cells were cultured with DMEM/F12 incomplete high-sugar medium containing $10 \%$ FBS in an atmosphere of $95 \%$ air and $5 \% \mathrm{CO}_{2}$ at $37^{\circ} \mathrm{C}$ for $24 \mathrm{~h}$. Alisma triterpenes were diluted by DMEM/F12 incomplete high-sugar medium to $1.0 \times 10^{-3}, 5 \times 10^{-4}, 2.5 \times 10^{-4}, 1.25 \times 10^{-4}, 6.25 \times 10^{-5}, 3.125 \times 10^{-5}$, $1.5625 \times 10^{-5}, 7.8125 \times 10^{-6} \mathrm{~g} / \mathrm{mL}$ concentrations for further experiments.

\subsection{Cell activity detection of HK-2 cells by MTT colorimetry}

After incubation, the cells were incubated with the final concentration of drug solution for $24 \mathrm{~h}$ in an atmosphere of $95 \%$ air and $5 \% \mathrm{CO}_{2}$ at $37^{\circ} \mathrm{C}$. The medium without drug solution was used as blank control. Then $0.5 \mathrm{mg} / \mathrm{mL}$ MTT $(100 \mu \mathrm{L})$ was added into each well of the media. The cells were incubated for four $\mathrm{h}$. Then $100 \mu \mathrm{L}$ of dimethyl sulfoxide (DMSO) was added into each well and vibrated at $37^{\circ} \mathrm{C}$ for $10 \mathrm{~min}$ to dissolve crystals. The absorbance optical density value of cells was measured in an automatic microplate reader (Thermo, USA). The surviving fraction of cells was calculated for each assay as the percentage of cell viability $=$ (optical density of components group - optical density of blank group) / (optical density of cell blank group - optical density of blank plate) $\times 100 \%$.

\section{Immunocytochemistry}

After being treated with drugs for $24 \mathrm{~h}, 4 \%$ formaldehyde solution was used to fix the cells for $24 \mathrm{~h}$ and the cells were placed in antigen repair buffer, boiled for $20 \mathrm{~min}$. The cells were dripped with $3 \% \quad \mathrm{H}_{2} \mathrm{O}_{2}$-PBS solution to block endogenous peroxidase at room temperature for fifteen min. Then the cells were blocked with $10 \%$ primary serum or $5 \%$ 
Table 1: The fragment information of triterpenes in alisma

\begin{tabular}{llllll}
\hline No. & Compound & $\mathrm{T}_{\mathrm{R}}(\mathrm{min})$ & $(+)$ ESI-MS m/z & $\mathrm{UV}_{\max }(\mathrm{nm})$ & $(+)$ ESI-MS m/z (ion fragments) \\
\hline 1 & 16-oxo-alisol A & 13.752 & 505.20 & 208,4 & $505.2,415.25$ \\
2 & 16-oxo-alisol A 24-actetate & 14.231 & 547.30 & 208,4 & $547.25,529.20,358.10$ \\
3 & 16-oxo-alisol A 23-actetate & 14.792 & 547.30 & 208,4 & \\
4 & Alisol C & 14.975 & 487.20 & 208,4 & $487.20,431.15,358.10$ \\
5 & Alisol C 23-acetate & 17.810 & 529.20 & 208,4 & $529.20,358.10$ \\
\hline
\end{tabular}

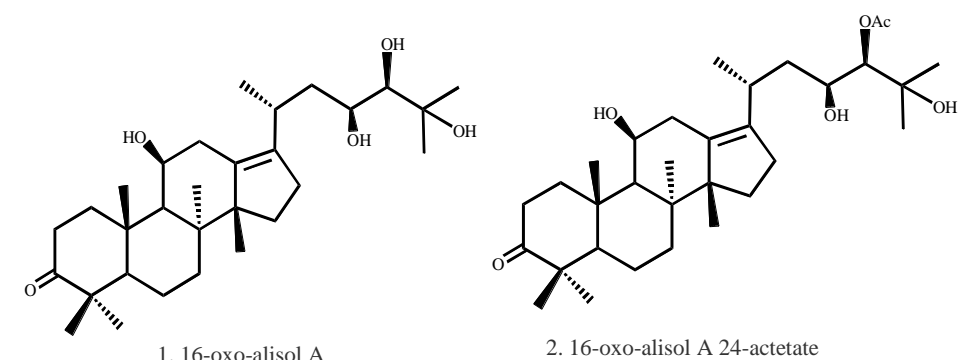

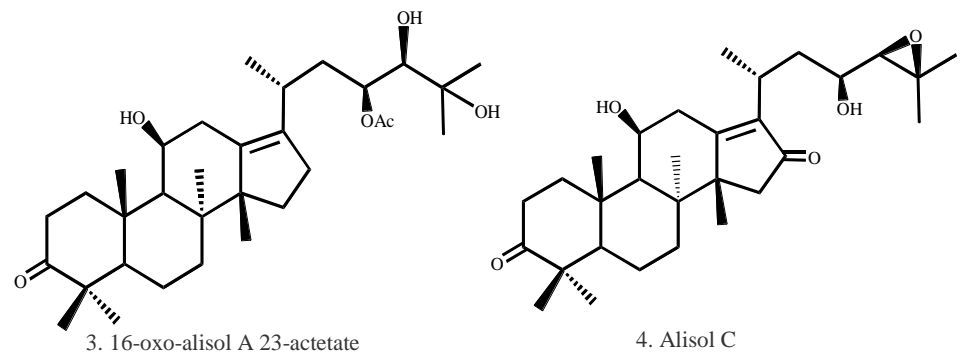

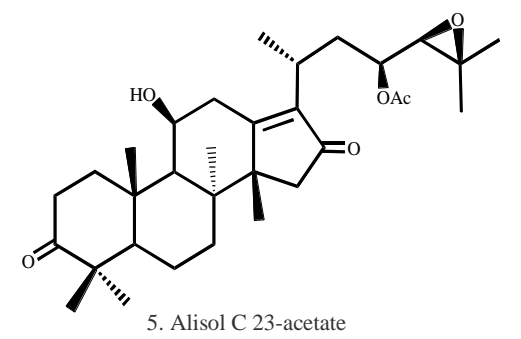

Fig. 1: The chemical structure of Alisma Triterpenes including 16-oxo-alisol A, 16-oxo-alisol A 24-actetate, 16-oxo-alisol A 23-actetate, Alisol C, Alisol C 23-acetate

BSA $\left(4^{\circ} \mathrm{C}\right.$, overnight). After being washed with PBS for 4 times, the sections were incubated with biotin-conjugated secondary antibody at $37^{\circ} \mathrm{C}$ for $30 \mathrm{~min}$. Appropriate amount of DAB solution was added on sections for staining and color development. The sections were redyed with hematoxylin staining solution for $10 \mathrm{~min}$ and rinsed with distilled water. After the cells drying, neutral gum was added on the section for sealing. Negative controls were incubated and processed identically with omission of the primary antibody. The expression of visualization was observed and photographed under a microscope.

\section{Statistical analysis}

In this study, the obtained data are shown as means \pm standard deviation (SD). S.P.S.S. 16.0 software (Chicago, I.L., United States) was used to analyze the statistical differences between two groups or among more than two groups using One-way analysis of variance (ANOVA) with Bonferroni post hoc test. A value of $P<0.05$ was regarded as statistically significant.

\section{Results}

\section{Analysis and determination for triterpenes in alisma}

In this study, high-performance liquid chromatographyquadrupole time-of-flight mass spectrometry (HPLCQ-TOF-MS) was used to analyze the triterpenes in alisma. The results showed that triterpenes in alisma contain mainly 16-oxo-alisol A, 16-oxo-alisol A 24actetate, 16-oxo-alisol A 23-actetate, Alisol C, Alisol C 23-acetate, and so on (Fig. 1). The detailed fragments information is listed in Fig. 2 and Table 1. 
Renal Regulation of Terpenoids Composition in Alisma / Intl J Agric Biol, Vol 25, No 2, 2021

Table 2: Diuretic activities of Alisma triterpenes on urine volume and urine $\mathrm{Na}^{+}, \mathrm{K}^{+}$and $\mathrm{Cl}^{-}$concentrations in rats (means $\pm \mathrm{SD}$, $\mathrm{n}=6$ ). Note: compared with the blank control group, $* * * P<0.001, * * P<0.01, * P<0.05$

\begin{tabular}{|c|c|c|c|c|c|}
\hline Groups & Dosage $(\mathrm{mg} / \mathrm{kg} / \mathrm{d})$ & Urine volume $(\mathrm{mL})$ & $\mathrm{Na}^{+}(\mathrm{mmol} / \mathrm{L})$ & $\mathrm{K}^{+}(\mathrm{mmol} / \mathrm{L})$ & $\mathrm{Cl}^{-}(\mathrm{mmol} / \mathrm{L})$ \\
\hline Blank control & - & $4.33 \pm 1.78$ & $19.55 \pm 1.91$ & $11.79 \pm 1.12$ & $20.7 \pm 2.83$ \\
\hline Furosemide & 20 & $15.75 \pm 1.81^{* * *}$ & $88.87 \pm 2.86^{* * *}$ & $28.91 \pm 15.08^{*}$ & $95.8 \pm 1.65^{* * * *}$ \\
\hline High-dose triterpenes & 80 & $11.00 \pm 1.04^{*}$ & $41.4 \pm 5.16^{* *}$ & $25.02 \pm 2.46^{*}$ & $36.93 \pm 7.32 *$ \\
\hline Medium-dose triterpenes & 40 & $9.63 \pm 1.53^{*}$ & $33.9 \pm 16.12$ & $20.66 \pm 9.72$ & $25.6 \pm 7.92$ \\
\hline Low-dose triterpenes & 20 & $7.50 \pm 3.81$ & $19.9 \pm 13.72$ & $19.58 \pm 3.93$ & $22.33 \pm 9.52$ \\
\hline
\end{tabular}
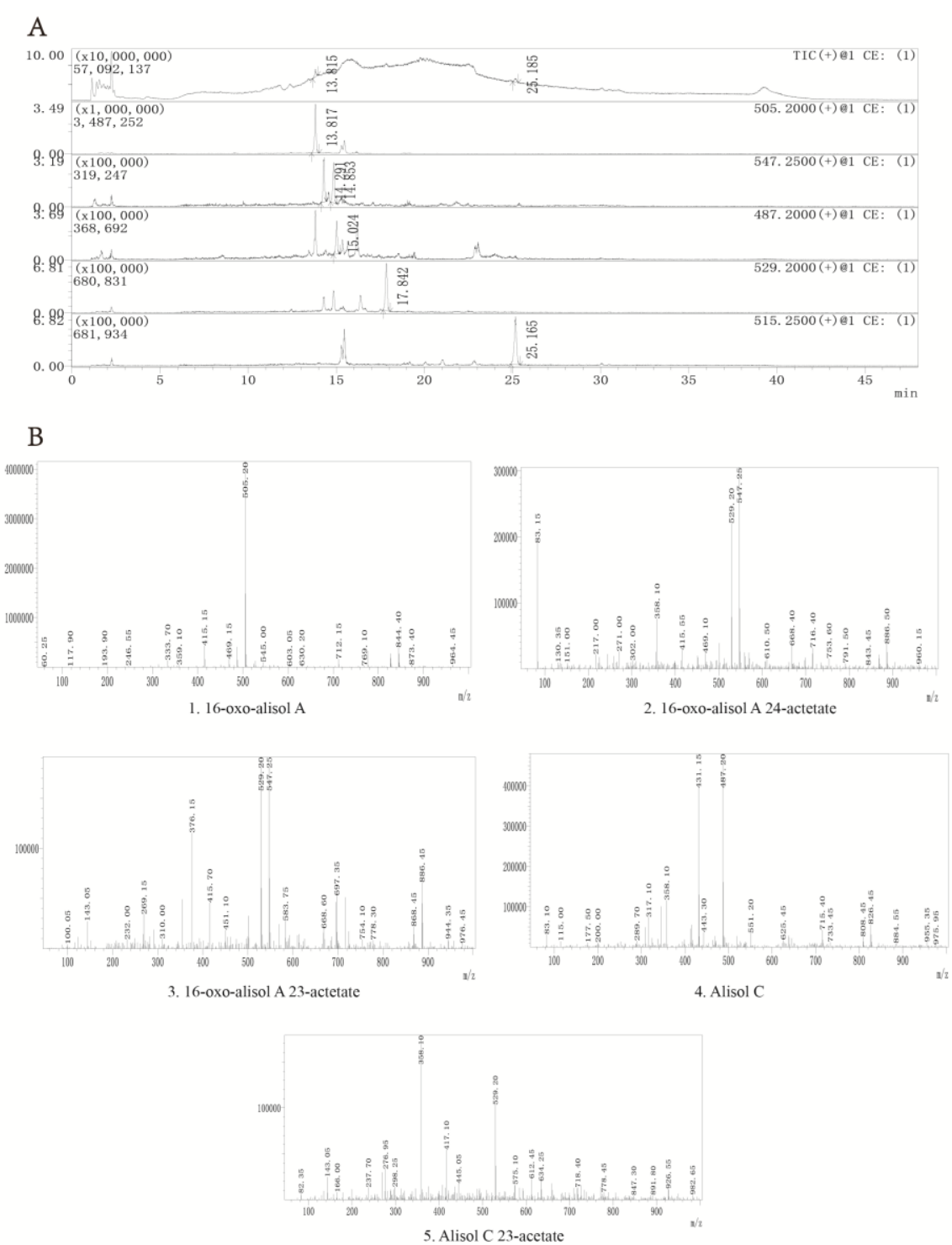

Fig. 2: MS ion flow diagram (A) and ion spectra (B)

\section{Alisma triterpenes increased urine volume and urine concentrations of $\mathrm{Na}^{+}, \mathrm{K}^{+}$and $\mathrm{Cl}^{-}$in water load model rats}

Compared with the blank control, positive control Furosemide significantly increased urine volume and the concentrations of $\mathrm{Na}^{+}, \mathrm{K}^{+}$and $\mathrm{Cl}^{-}$in urine $(P<$ 0.001 or $P<0.05)$. Interestingly, the treatment of medium-dose triterpene group $(40 \mathrm{mg} / \mathrm{kg} / \mathrm{d})$ and high- dose triterpene group $(80 \mathrm{mg} / \mathrm{kg} / \mathrm{d})$ enhanced urine volume remarkably when compared with the blank group. Similarly, urine $\mathrm{Na}^{+}, \mathrm{K}^{+}$and $\mathrm{Cl}^{-}$concentrations were also significantly increased by the treatment with triterpene of $80 \mathrm{mg} / \mathrm{kg} / \mathrm{d}(P<0.05)$ (Fig. 3 and Table 2). These results suggested that alisma triterpenes could increase the urine volume and promote the excretion of electrolytes in the urine of water - stressed rats, showing a certain diuretic effect. 

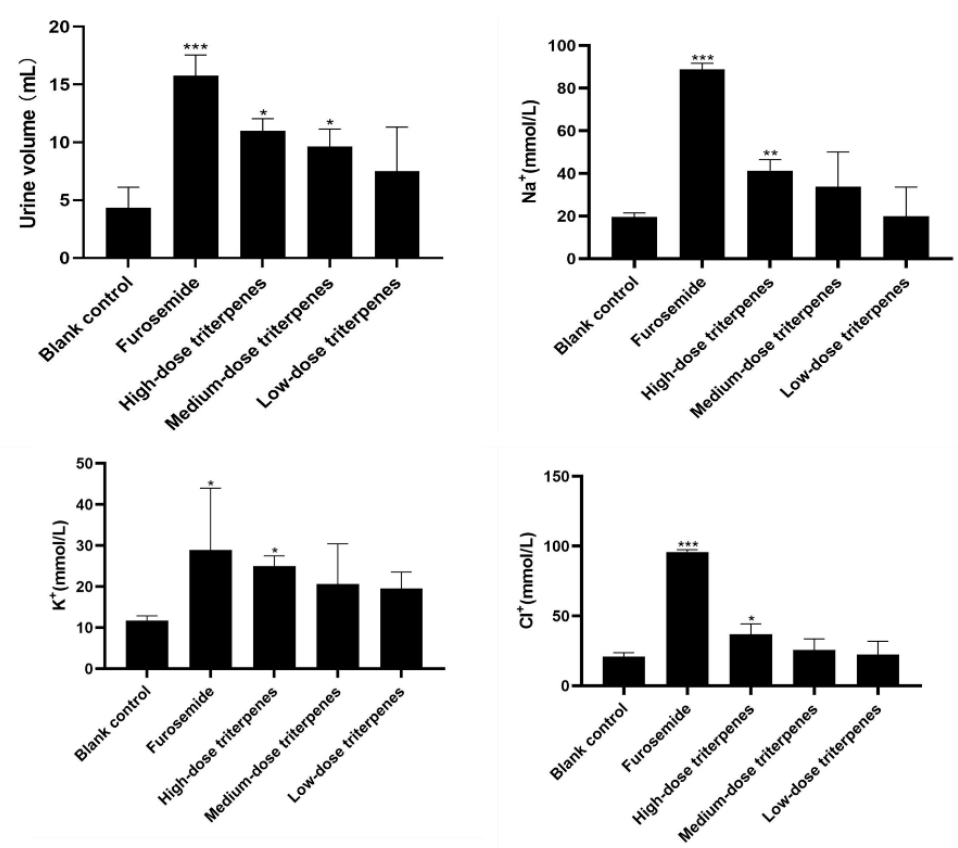

Fig. 3: Effects of Alisma triterpenes on the urine volume and the excretion of electrolytes in the urine of water - stressed rats (means $\pm S D, n=6$ ) A. The urine volume in the urine of water - stressed rats; $\mathbf{B}$. The excretion of $\mathrm{Na}^{+}$in the urine of water - stressed rats; $\mathbf{C}$. The excretion of $\mathrm{K}^{+}$in the urine of water - stressed rats; $\mathbf{D}$ The excretion of $\mathrm{Cl}^{-}$in the urine of water - stressed rats. ${ }^{* * *} P<0.001, * * P<0.01, * P<0.05$ compared with the blank control group

A

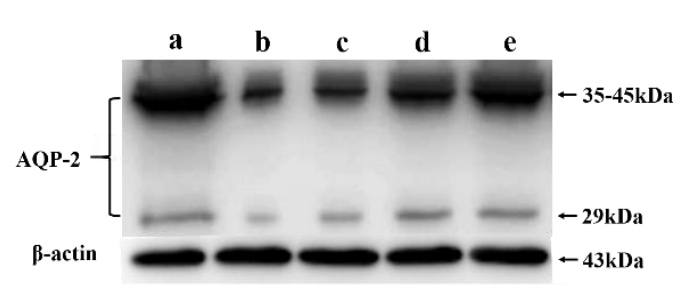

B

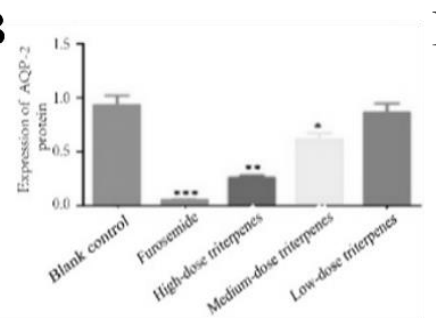

D

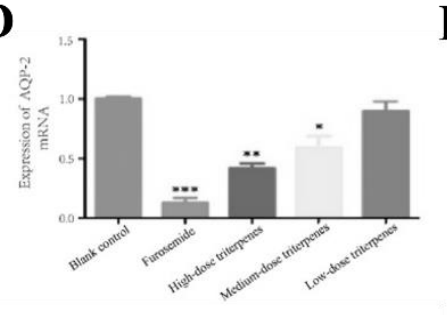

C

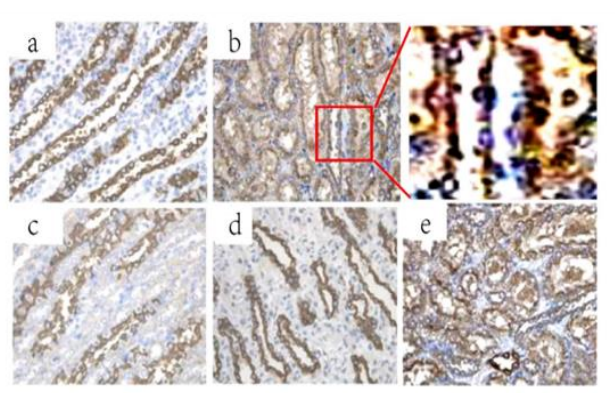

$\mathbf{E}$

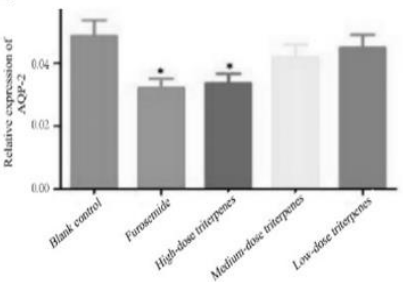

Fig. 4: Effects of Alisma triterpenes on AQP-2 in rats kidney (means $\pm S D, n=6$ )

A. The examination of AQP-2 level in kidney tissue by western blotting; B. The examination of AQP-2 mRNA level in kidney tissue by RT-PCR; C. The relative expression of AQP-2 in kidney tissue by immunochemistry. a, b, c, d, e respectively represent blank control group, furosemide group, high-dose triterpenes group, medium-dose triterpenes group and low-dose triterpenes group; ${ }^{* * * *} P<0.001,{ }^{*} P<0.01,{ }^{*} P<0.05$, compared with the blank control group

Effects of alisma triterpenes on the expression of AQP-2 protein and mRNA in rat kidney

As shown in Fig. 4A and 4C, both Western Blot and Immunochemistry results showed that a significant reduction on AQP-2 expression in rat kidney tissue treated with alisma triterpenes, especially in high-dose group and medium-dose group $(P<0.01$ or $P<0.05)$.
Furthermore, RT-PCR results showed that the expression of AQP-2 mRNA in renal medullary was significantly reduced in furosemide, high-dose triterpenes and medium-dose triterpenes groups, when compared with blank group (Fig. 4B; $P<0.01$ or $P<$ $0.05)$. Therefore, it could be concluded that alisma triterpenes could reduce the expression level of renal $\mathrm{AQP}-2$ in rats. 
Table 3: Cell viability of different concentration of ZTCs in HK-2 cells (means \pm SD, $n=6$ )

\begin{tabular}{lll}
\hline Groups & Dosage $(\mathrm{g} / \mathrm{mL})$ & Relative cell survival rate $(\%)$ \\
\hline Blank control & - & 100 \\
Triterpenes & $7.8125 \times 10^{-6}$ & $96.13 \pm 4.37$ \\
& $1.5625 \times 10^{-5}$ & $95.37 \pm 2.86$ \\
& $3.125 \times 10^{-5}$ & $94.64 \pm 3.91$ \\
& $6.25 \times 10^{-5}$ & $92.97 \pm 2.41$ \\
$1.25 \times 10^{-4}$ & $91.24 \pm 3.83$ \\
$2.5 \times 10^{-4}$ & $92.75 \pm 2.30$ \\
& $5.0 \times 10^{-4}$ & $90.52 \pm 3.11$ \\
& $1.0 \times 10^{-3}$ & $86.86 \pm 1.91$ \\
\hline
\end{tabular}

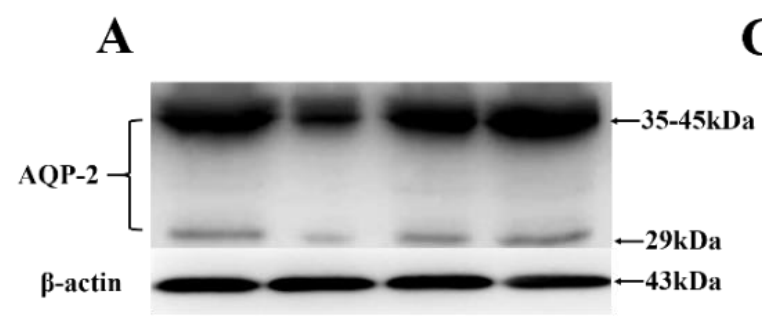

B

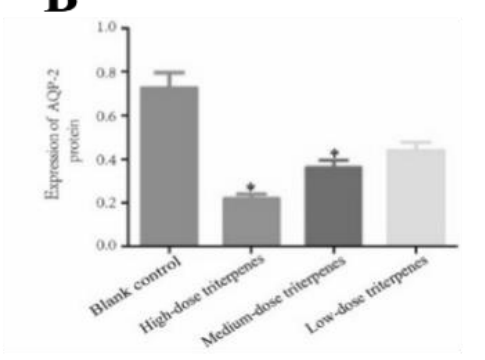

D

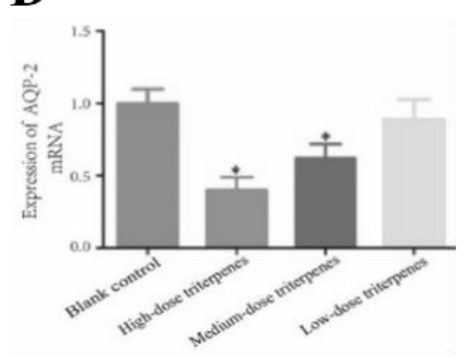

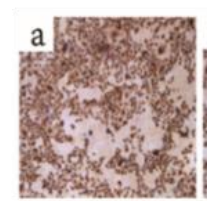
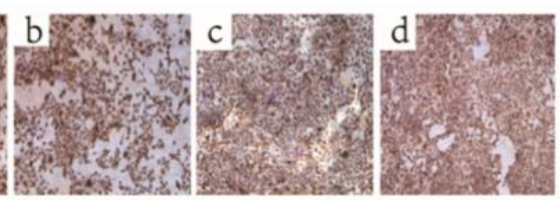

E

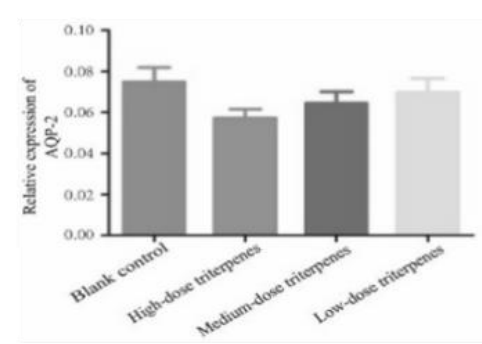

Fig. 5: Effects of Alisma triterpenes on AQP-2 in HK-2 cells (means $\pm S D, n=6$ )

$\mathbf{A}$ and $\mathbf{B}$. The examination of AQP-2 level in HK-2 cells by western blotting

C and $\mathbf{E}$. The relative expression of AQP-2 in HK-2 cells by immunochemistry. a, b, c, d, respectively represent blank control group, high-dose triterpenes group, medium-dose triterpenes group and low-dose triterpenes group

D. The examination of AQP-2 mRNA level in HK-2 cells by RT-PCR; ${ }^{*} P<0.05$, compared with blank control group

\section{Optimal concentration for alisma triterpenes to survival rate of HK-2 cells}

In order to explore the regulation of alisma triterpenes on AQP-2 in vitro, the suitable concentration, namely, minimum concentration affecting cell viability. When the cell survival rate is greater than $95 \%$, the drug is considered to be non-toxic to cells. MTT results showed that the cell survival rate was $(95.37 \pm 2.86 \%)$, indicating that alisma triterpenes has no obvious cytotoxicity to $\mathrm{HK}-2$ cells at this concentration (Table 3). Therefore, $3.125 \times 10^{-5} \mathrm{~g} / \mathrm{mL}$, $1.5625 \times 10^{-5} \mathrm{~g} / \mathrm{mL}$ and $7.8125 \times 10^{-6} \mathrm{~g} / \mathrm{mL}$ were used for subsequent experimental studies.

\section{The regulation of Alisma triterpenes on AQP-2 in HK-2 cells}

As shown in Fig. 5A and 5B, the expression of AQP-2 in cells was significantly decreased in the high-dose and medium-dose triterpenes groups $\left(3.125 \times 10^{-5} \mathrm{~g} / \mathrm{mL}, 1.5625 \times\right.$ $\left.10^{-5} \mathrm{~g} / \mathrm{mL}\right)(P<0.05)$ when compared with the blank control.
RT-PCR results (Fig. 5D) showed that the relative expression level of AQP-2 mRNA was also been reduced by the treatment of high-dose triterpenes $(P<0.05)$. In immunocytochemistry experiment, a significant reduction on AQP-2 positive brown deposit can be seen in triterpenestreated cells at the concentration of high-dose and mediumdose (Fig. 5C and 5E). The phenomena in vitro experiments further suggested that the regulation of AQP-2 protein and mRNA contributed to the diuretic effect of alisma terpenoids.

\section{Discussion}

Modern pharmacological studies showed that alisma has a variety of biological activities, such as diuretics, antisteatotic, antioxidant, antilipoapoptotic, hepatoprotective, anti-inflammatory, antifibrotic, antiobesity, hypoglycemic and hypolipidemic activities (Shu et al. 2016; Choi et al. 2019). Many studies were conducted on the isolation and pharmacological activities of triterpenes and sesquiterpenes, which are the main and most significant components of alisma (Ma et al. 2016). Despite ongoing studies, 
pharmacodynamic effects and mechanisms of constituents in alisma have still not revealed. This study focused on looking for the pharmacodynamic effects and mechanisms of triterpenes in alisma.

Aquaporin (AQP-2) is the main mechanism protein of diuretic traditional Chinese medicine, regulating the body's water balance (Xiong et al. 2019). Based on the theory of "component structure", this study explored the diuretic effect and molecular mechanism of terpenoids, which are the main active components in alismatis rhizoma. The diuretic effects of terpenoids in alisma were investigated by in vivo animal experiments and in vitro cell experiments respectively, and $\mathrm{WB}, \mathrm{PCR}$ and $\mathrm{IHC}$ were used to detect the expression level of AQP-2 protein in kidney of rats after administration. The experimental results showed that terpenoids in alisma can increase urine volume, promote $\mathrm{Na}^{+}, \mathrm{K}^{+}$, and $\mathrm{Cl}^{-}$excretion in urine in urine, reduce the expression of AQP-2, and exert the diuretic effect. This indicated that the molecular mechanism of the diuretic effect is achieved by reducing the expression of renal AQP-2.

Aquaporin is located in the collecting duct and is regulated by antidiuretic hormone. AVP triggers the redistribution of AQP-2 from intracellular vesicles (Schrade et al. 2018). AVP is released from the posterior lobe of the neurohypophysis and promotes the translocation or transport of AQP-2 from intracellular vesicles of the cells. From vivo animal experiments and vitro cell experiments, we found that terpenoids can achieve diuretic effect by regulating renal aquaporins. It is concluded that terpenoids reduce the expression of aquaporin by reducing the secretion of AVP.

\section{Conclusion}

At present, research on pharmacology and toxicology of alisma has made great progress. But there are still some problems, with modern pharmacology and toxicology research methods and related scientific research and technology development, alisma pharmacology and toxicology studies will get further development and perfection, these studies can provide more safe and reasonable clinical application of alisma guidance and contribution, also can better promote the development of alisma resources in clinical application.

\section{Acknowledgements}

The author sincerely acknowledges the support of these funds for the research of this project: National Natural Science Foundation of P.R. China (No. 81603382), Key research projects on modernization of traditional Chinese medicine (2018YFC1706900), "333 Project" research projects of Jiangsu province (BRA5475); and "Double FirstClass" University project of China Pharmaceutical University (CPU2018GF07, CPU2018PZQ19).

\section{Author Contributions}

Important contributions to design and also to prepare this manuscript: X-BJ applied for the grant, conceived and designed this study. L.F. prepared the experiment and designed this study. CL, Z-LL, X-HL and Q-LL, performed all of the experiments. S-TP analyzed the data and wrote the manuscript. All authors participated in the preparation of the manuscript and approved the final version.

\section{References}

Choi E, E Jang, JH Lee (2019). Pharmacological activities of Alisma orientale against nonalcoholic fatty liver disease and metabolic syndrome: Literature review. Evid Based Complement Altern Med 2019; Article 2943162

Huang J, JP Montani, F Verrey, E Feraille, XF Ming, Z Yang (2018). Arginase-II negatively regulates renal aquaporin-2 and water reabsorption. FASEB J 32:5520-5531

Jeong HS, YH Cho, KH Kim, Y Kim, KS Kim, YC Na, J Park, IS Lee, JH Lee, HJ Jang (2016). Anti-lipoapoptotic effects of Alisma orientalis extract on non-esterified fatty acid-induced HepG2 cells. BMC Complement Altern Med 16; Article 239

Jung HJ, TH Kwon (2016). Molecular mechanisms regulating aquaporin-2 in kidney collecting duct. Amer J Physiol Renal Physiol 311:13181328

Ma Q, L Han, X Bi, X Wang, Y Mu, P Guan, L Li, X Huang (2016). Structures and biological activities of the triterpenoids and sesquiterpenoids from Alisma orientale. Phytochemistry 131:150157

Mamuya FA, JL Cano-Peñalver, W Li, D Rodriguez Puyol, M Rodriguez Puyol, D Brown, S de Frutos, HA Lu (2016). ILK and cytoskeletal architecture: An important determinant of AQP2 recycling and subsequent entry into the exocytotic pathway. Amer J Physiol Renal Physiol 311:1346-1357

Mose FH, AN Jörgensen, MH Vrist, NP Ekelöf, EB Pedersen, JN Bech (2019). Effect of 3\% saline and furosemide on biomarkers of kidney injury and renal tubular function and GFR in healthy subjects - a randomized controlled trial. BMC Nephrol 20; Article 200

Schrade K, J Tröger, A Eldahshan, K Zühlke, KR Abdul Azeez, JM Elkins, M Neuenschwander, A Oder, M Elkewedi, S Jaksch, K Andrae, J Li, J Fernandes, PM Müller, S Grunwald, SF Marino, T Vukićević, J Eichhorst, B Wiesner, M Weber, M Kapiloff, O Rocks, O Daumke, T Wieland, S Knapp, JPV Kries, E Klussmann (2018). An AKAP-Lbc-RhoA interaction inhibitor promotes the translocation of aquaporin-2 to the plasma membrane of renal collecting duct principal cells. PLoS One 13; Article e0191423

Shu Z, J Pu, L Chen, Y Zhang, K Rahman, L Qin, C Zheng (2016). Alisma orientale: Ethnopharmacology, Phytochemistry and Pharmacology of an Important Traditional Chinese Medicine. Amer J Chin Med 44:227-51

Xiong R, Y Li, K Zheng, T Zhang, M Gao, Y Li, Y Lian, D Chen, C Hu (2019). Er Shen Wan extract alleviates polyuria and regulates AQP 2 and AVPR 2 in a rat model of spleen-kidney Yang deficiencyinduced diarrhea. Biomed Pharmacother 110:302-311

Zhang LL, W Xu, YL Xu, X Chen, M Huang, JJ Lu (2017). Therapeutic potential of Rhizoma Alismatis: A review on ethnomedicinal application, phytochemistry, pharmacology, and toxicology. Ann NY Acad Sci 1401:90-101

Zhang X, XY Li, N Lin, WL Zhao, XQ Huang, Y Chen, MQ Huang, W Xu, SS Wu (2017). Diuretic Activity of Compatible Triterpene Components of Alismatis rhizoma. Molecules 22; Article 1459 